\title{
Three-dimensional angular momentum projected relativistic point-coupling approach for the low-lying excited states in ${ }^{24} \mathrm{Mg}$
}

\author{
J. M. Yao, ${ }^{1,2, *}$ J. Meng, $, 1,3,4, \dagger$ D. Pena Arteaga, ${ }^{2}$ and P. Ring ${ }^{2}$ \\ ${ }^{1}$ School of Physics and State Key Laboratory of Nuclear Physics and Technology, \\ Peking University, 100871 Beijing, China \\ ${ }^{2}$ Physik-Department der Technischen Universität München, D-85748 Garching, Germany \\ ${ }^{3}$ Institute of Theoretical Physics, Chinese Academy of Sciences, Beijing, China \\ ${ }^{4}$ Center of Theoretical Nuclear Physics, \\ National Laboratory of Heavy Ion Accelerator, 730000 Lanzhou, China
}

(Dated: November 3, 2018)

\begin{abstract}
A full three-dimensional angular momentum projection on top of a triaxial relativistic mean-field calculation has been implemented for the first time. The underlying Lagrangian is a point coupling model and pairing correlations are taken into account by a monopole force. This method is applied for the low-lying excited states in ${ }^{24} \mathrm{Mg}$. Good agreement with the experimental data is found for the ground state properties. A minimum in the potential energy surface for the $2_{1}^{+}$state, with $\beta \simeq 0.55, \gamma \simeq 10^{\circ}$, is used as the basis to investigate the rotational energy spectrum as well as the corresponding $\mathrm{B}(\mathrm{E} 2)$ transition probabilities as compared to the available data.
\end{abstract}

PACS numbers: 21.10.Dr, 21.10.Re, 21.60.Jz, 21.30.Fe

*Electronic address: jmyao@pku.edu.cn

${ }^{\dagger}$ Electronic address: mengj@pku.edu.cn 
One of the most discussed topics since the 60's has been the possible triaxial nature of $2 \mathrm{~s}-1 \mathrm{~d}$ shell nuclei. The best candidate to show such a deformation is the nucleus ${ }^{24} \mathrm{Mg}$, which has been extensively studied both experimentally and with state-of-the-art theoretical models. However, the question of whether it is triaxial is still not well settled.

Early experiments and their interpretation, such as the spectroscopic factors extracted from the ${ }^{24} \mathrm{Mg}(\mathrm{d}, \mathrm{p})^{25} \mathrm{Mg}$ reaction data done by Parikh [1], favor a prolate axial deformation. On the other hand, there is also evidence that supports the triaxiality of ${ }^{24} \mathrm{Mg}$, like the interpretation of inelastic polarized proton scattering by Lombard et al. [2], or the existence of a low-lying $K=2$ band [3]. However, the interpretation of this band as a rotational band or a band built on one-phonon $\gamma$-vibrational states, is the origin of much controversy. Phenomenologically, the energy spectrum for the low-lying states of ${ }^{24} \mathrm{Mg}$ is well described by the asymmetric rotor model of Davydov-Filipov with a triaxiality parameter $\gamma=22^{\circ}$, while the branching ratios and the reduced transition rates are not [4]. Furthermore, the interband transitions are reproduced with $\gamma=14^{\circ}$, a value that cannot give the correct energy spectrum [5].

Many microscopic studies have been done to describe the shape of ${ }^{24} \mathrm{Mg}$. So far, most of them have relied on investigations of the potential energy surface (PES) within various mean-field approaches [6]. Early triaxial Hartree-Fock (HF) calculations with different Skyrme forces showed that the shape depends strongly on the spin-orbit part of the interaction [7]. With a proper treatment of this term, both Hartree-Fock-Bogoliubov (HFB) calculations with the Gogny force [8] as well as HF+BCS investigations with the Skyrme force [9], yield axially symmetric prolate minima. However, a Three Dimensional (3D) cranked HFB calculation within the pairing-plus-quadrupole model predicts a substantial triaxial deformation for states with higher angular momenta $J>4$ in the ground state band [10]. Similar investigations have been also carried out within the relativistic meanfield (RMF) approach, and an axial prolate ground state has been predicted for ${ }^{24} \mathrm{Mg}$ with either NL1 [11] or TM1 [12] parameter sets.

All the mentioned microscopic studies are based on the mean-field approximation, which is able to take into account correlations by "spontaneous symmetry breaking", e.g., by the introduction of a deformed mean-field that breaks rotational symmetry in the intrinsic frame. In order to compare properly with the experimental data, one has to transform to the laboratory system and to resort to Angular Momentum Projection (AMP) to restore the 
broken symmetry. However, due to its very demanding numerical nature, only very recently it has been possible to apply such a projection procedure to study the low-lying excited states of ${ }^{24} \mathrm{Mg}$, in the context of the Generator Coordinate Method (GCM), for example with the Skyrme SLy4 force [13], the Gogny D1S force [14] and the relativistic point-coupling model with PC-F1 force [15]. These investigations have shown that the energy gain due to the restoration of rotational symmetry is of the order of several MeV. Meanwhile, it has to be pointed out that all these AMP+GCM studies are restricted to axially symmetric meanfields. Although great success was achieved in the description of both the spectrum and $\mathrm{B}(\mathrm{E} 2)$ transition probabilities with $J<6$, neither the triaxial deformation information nor a satisfactory description of the observed $K=2$ band could be obtained. Therefore, it is essential to introduce triaxial quadrupole deformation at the mean-field level and perform full three-dimensional angular momentum projection (3DAMP).

In the context of the phenomenological models, 3DAMP has been done using small shell model spaces and the corresponding effective interactions [16, 17, 18, 19]. It is shown that the restoration of rotational symmetry has a strong influence on the topological structure of $(\beta, \gamma)$ energy surface [19]. In the context of energy density functionals, 3DAMP has been performed on top of Hartree-Fock with a simple Skyrme-type interaction [20], or with a full Skyrme energy functional [21]. In both cases cranked wave functions were projected to approximate a variation after projection procedure, but pairing correlations were not included. Only very recently, projection on particle number (PNP) and angular momentum with configuration mixing has been attempted in a triaxial HFB theory for the study the low-lying excited states of ${ }^{24} \mathrm{Mg}[22]$.

RMF theory, which relies on the ideas of effective field theory and of density functional theory, represents a very successful approach to low energy nuclear structure studies with a few universal parameters [23, 24, 25, 26]. It incorporates many important relativistic effects from the beginning, such as the presence of large Lorentz scalar and vector mean fields with approximately equal magnitude and opposite sign that leads to a new saturation mechanism via the difference between the scalar and vector densities, and yields naturally the large spin-orbit splitting needed for the understanding of magic numbers in finite nuclei. In this manuscript, for the first time, 3DAMP has been implemented on top of triaxial relativistic mean-field theory based on point-coupling (RMF-PC) interactions, and will be applied in this Letter to study the low-lying excited states of ${ }^{24} \mathrm{Mg}$. 
In this approach, the intrinsic mean-field state is obtained through the minimization of an energy functional based on the following Lagrangian density [27]

$$
\begin{aligned}
\mathcal{L}= & \bar{\psi}\left(i \gamma_{\mu} \partial^{\mu}-m\right) \psi \\
& -\frac{1}{2} \alpha_{S}(\bar{\psi} \psi)(\bar{\psi} \psi)-\frac{1}{2} \alpha_{V}\left(\bar{\psi} \gamma_{\mu} \psi\right)\left(\bar{\psi} \gamma^{\mu} \psi\right) \\
& -\frac{1}{2} \alpha_{T S}(\bar{\psi} \vec{\tau} \psi) \cdot(\bar{\psi} \vec{\tau} \psi)-\frac{1}{2} \alpha_{T V}\left(\bar{\psi} \vec{\tau} \gamma_{\mu} \psi\right) \cdot\left(\bar{\psi} \vec{\tau} \gamma^{\mu} \psi\right) \\
& -\frac{1}{3} \beta_{S}(\bar{\psi} \psi)^{3}-\frac{1}{4} \gamma_{S}(\bar{\psi} \psi)^{4}-\frac{1}{4} \gamma_{V}\left[\left(\bar{\psi} \gamma_{\mu} \psi\right)\left(\bar{\psi} \gamma^{\mu} \psi\right)\right]^{2} \\
& -\frac{1}{2} \delta_{S} \partial_{\nu}(\bar{\psi} \psi) \partial^{\nu}(\bar{\psi} \psi)-\frac{1}{2} \delta_{V} \partial_{\nu}\left(\bar{\psi} \gamma_{\mu} \psi\right) \partial^{\nu}\left(\bar{\psi} \gamma^{\mu} \psi\right) \\
& -\frac{1}{2} \delta_{T S} \partial_{\nu}(\bar{\psi} \vec{\tau} \psi) \cdot \partial^{\nu}(\bar{\psi} \vec{\tau} \psi) \\
& -\frac{1}{2} \delta_{T V} \partial_{\nu}\left(\bar{\psi} \vec{\tau} \gamma_{\mu} \psi\right) \cdot \partial^{\nu}\left(\bar{\psi} \vec{\tau} \gamma^{\mu} \psi\right) \\
& -\frac{1}{4} F^{\mu \nu} F_{\mu \nu}-e \frac{1-\tau_{3}}{2} \bar{\psi} \gamma^{\mu} \psi A_{\mu} .
\end{aligned}
$$

which contains eleven coupling constants, $\alpha_{S}, \alpha_{V}, \alpha_{T S}, \alpha_{T V}, \beta_{S}, \gamma_{S}, \gamma_{V}, \delta_{S}, \delta_{V}, \delta_{T S}$ and $\delta_{T V}$, where the subscript indicates the symmetry of the coupling: $S$ stands for scalar, $V$ for vector and $T$ for isovector, while the Greek letters indicate the kind of contact interaction: $\alpha$ refers to four-fermion terms, $\beta$ and $\gamma$ to third- and fourth-order terms respectively, and $\delta$ to derivative couplings.

From the Lagrangian density in Eq.(1), one can easily obtain an energy density functional, whose minimization provides the intrinsic mean-field state wave-function. So far, pairing correlations have been taken into account within the BCS approach based on a monopole force and a smooth cutoff factor is taken into account to simulate the effects of finite range [28]. To obtain the PES, the mass quadrupole moments are constrained through the quantities $q_{20}$ and $q_{22}$, which are related to the triaxial deformation parameters $\beta$ and $\gamma$ of the Bohr Hamiltonian by $q_{20}=\frac{3 A}{4 \pi} R_{0}^{2} \beta \cos \gamma$ and $q_{22}=\frac{3 A}{4 \pi} R_{0}^{2} \frac{1}{\sqrt{2}} \beta \sin \gamma$, where $R_{0}=1.2 A^{1 / 3}$ fm. The total mass quadrupole moment $q$ is thus given by $q=\sqrt{16 \pi / 5} \sqrt{q_{20}^{2}+2 q_{22}^{2}}$. During minimization, parity, $D_{2}$ symmetry, and time-reversal symmetry are imposed. The densities are thus symmetric with respect to reflections on the $x=0, y=0$ and $z=0$ planes. The parameter set chosen for the Lagrangian density in Eq.(11) is PC-F1 [27]. The solution of the equations of motion obtained from the energy density functional is accomplished by expansion on a set of isotropic three-dimensional harmonic oscillator basis in Cartesian coordinates with $N_{\mathrm{sh}}=8$ major shells. The oscillator length is chosen to be $b_{0}=\sqrt{\hbar / m \omega_{0}}$ 
with $\hbar \omega_{0}=41 A^{1 / 3} \mathrm{MeV}$. The pairing strength constants $G_{n}=34.60 / A$ and $G_{p}=33.75 / A$ are determined separately for neutrons and protons by adjusting the pairing gaps at the minimum of mean-field PES to the odd-even mass difference as obtained with a five-point formula, and kept fixed throughout the constraint calculation.

The wavefunction $\left|\Psi_{\alpha, q}^{J M}\right\rangle$ with good quantum numbers $\hat{J}$ and $\hat{J}_{z}$ has the form,

$$
\left|\Psi_{\alpha, q}^{J M}\right\rangle=\sum_{K \geq 0} f_{\alpha}^{J K}(q)|J M K+, q\rangle
$$

where the sum is restricted to non-negative even values of $K$. The index $\alpha=1,2, \cdots$ labels the different collective excitations. The angular momentum projected $K$-component, $|J M K+, q\rangle$, is given by

$$
|J M K+, q\rangle=\frac{1}{1+\delta_{K 0}}\left[\hat{P}_{M K}^{J}+(-1)^{J} \hat{P}_{M-K}^{J}\right]|\Phi(q)\rangle
$$

where the projection operator $\hat{P}_{M K}^{J}$ is defined as [6]

$$
\hat{P}_{M K}^{J}=\frac{2 J+1}{8 \pi^{2}} \int d \Omega D_{M K}^{J *}(\Omega) \hat{R}(\Omega)
$$

with $\Omega$ representing the set of three Euler angles. $D_{M K}^{J}(\Omega)$ is the corresponding Wigner function. The expansion coefficients $f_{\alpha}^{J K}(q)$ are determined by the generalized eigenvalue equation,

$$
\sum_{K^{\prime} \geq 0}\left\{\mathcal{H}_{K K^{\prime}}^{J}(q ; q)-E_{\alpha}^{J} \mathcal{N}_{K K^{\prime}}^{J}(q ; q)\right\} f_{\alpha}^{J K^{\prime}}(q)=0
$$

where the overlap kernels $(\mathcal{O}=\mathcal{N}, \mathcal{H})$ are given by:

$$
\mathcal{O}_{K K^{\prime}}^{J}(q ; q)=\left\langle J M K+q|\hat{O}| J M K^{\prime}+q\right\rangle, \quad \hat{O}=1, \hat{H}
$$

The overlap kernels are determined with the help of generalized Wick's theorem [29, 30]. In addition the method of Neergard and Wüst [31] is also used to determine the phase of the overlaps $\langle\hat{R}(\Omega)\rangle$, and it is found that both methods are in good agreement. The norm overlaps for intrinsic mean-field states with deformation $\gamma=0$ have been compared with those computed in the Gaussian overlap approximation, which turns out to be a good approximation, as already pointed out in Ref. [15]. This provides a very useful test of the numerical implementation of the angular momentum projection techniques. For the Hamiltonian overlap $\langle\hat{H} \hat{R}(\Omega)\rangle$, transition (or mixed) density matrices are adopted. The numerical evaluation of the kernels (6) is carried out by a N-point Gauss-Legendre quadrature over 
the Euler angles $\Omega=(\phi, \theta, \psi)$. With $N_{\theta}=14, N_{\phi}=N_{\psi}=24$, it is possible to achieve a precision of $0.001 \%$ in the energy of a projected state with an angular momentum up to $J=6$. With the consideration of symmetries in the overlaps [16, 18], only 1/16 of the total overlaps needs to be evaluated directly.

The standard procedure described in Ref. [6] has been used to solve the generalized eigenvalue equation (51). By construction, the collective wave function Eq.(2) avoids the appearance of zero eigenvalues, and therefore there are $J / 2+1$ or $(J-1) / 2$ collective states and rotational energy levels for even or odd angular momentum $J$ [18], respectively. These levels can be classified into different bands according to their B(E2) transition probabilities,

$$
B\left(E 2 ; q, J_{i}, \alpha_{i} \rightarrow q, J_{f}, \alpha_{f}\right)=\frac{e^{2}}{2 J_{i}+1}\left|\left\langle J_{f}, q\left\|\hat{Q}_{2}\right\| J_{i}, q\right\rangle\right|^{2}
$$

Since these quantities are calculated in full configuration space, there is no need to introduce effective charges, and hence $e$ denotes the bare value of proton charge. A full detailed discussion about the 3DAMP+RMF-PC approach can be found in Ref. [32].

Figure 1 shows the PES of ${ }^{24} \mathrm{Mg}$ in the $(\beta, \gamma)$ deformation plane, calculated with triaxial RMF-PC+BCS. The minimum with $E=-193.57 \mathrm{MeV}$, and $\beta_{p}=0.48$ is found which is less bound while compared with the data $E=-198.26 \mathrm{MeV}$ [33].

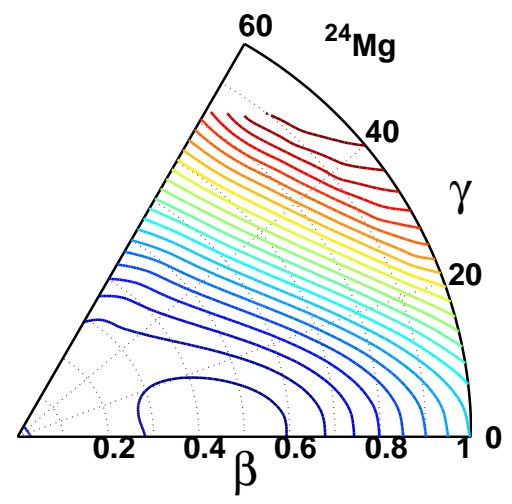

FIG. 1: (Color online) The potential energy surface in $\beta-\gamma$ plane obtained by triaxial RMF$\mathrm{PC}+\mathrm{BCS}$ calculation for ${ }^{24} \mathrm{Mg}$. The contour lines are separated by $1.0 \mathrm{MeV}$.

The PES of the first $J^{\pi}=2^{+}$and $J^{\pi}=0^{+}$projected states for ${ }^{24} \mathrm{Mg}$ in the $\beta$ - $\gamma$ plane are plotted in Fig. 2. There is no pronounced minimum with an obvious $\gamma$-deformation in the PES for the $0^{+}$state, which is in disagreement with the results of ref.[22], where a calculation with 3DAMP+PNP based on non-relativistic Skyrme HFB shows a pronounced 


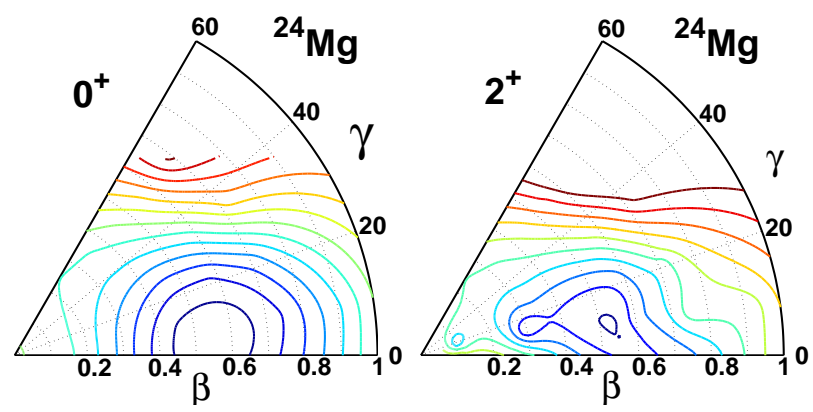

FIG. 2: (Color online) Three-dimensional angular momentum projected energy surfaces with $J=0$ (left panel) and $J=2$ (right panel) in the $\beta-\gamma$ plane for ${ }^{24} \mathrm{Mg}$. The contour lines are separated by $1.0 \mathrm{MeV}$
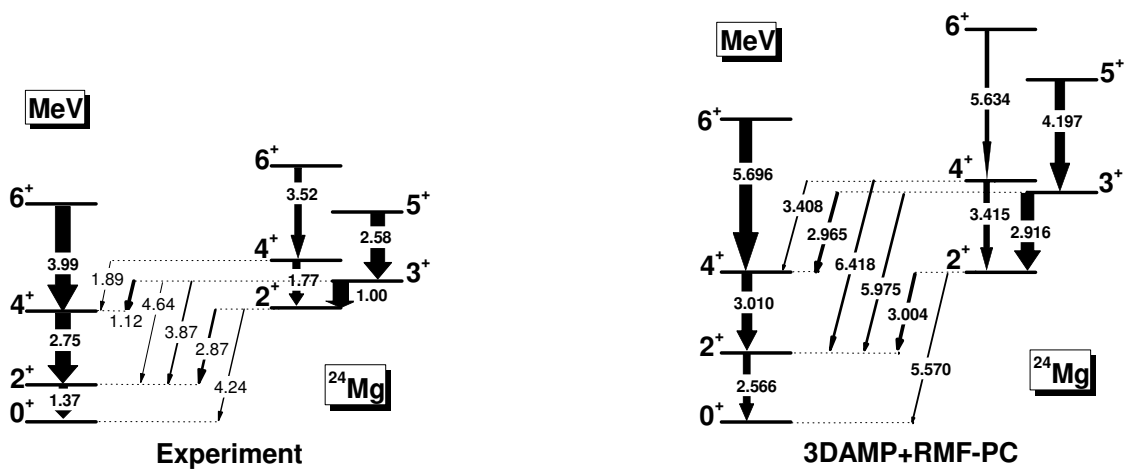

FIG. 3: Comparison between the theoretical and experimental bands in ${ }^{24} \mathrm{Mg}$. The excitation energies are normalized to the ground state and given in $\mathrm{MeV}$. The widths of the arrows represent the $B(E 2 \downarrow)$ values. The experimental data is taken from Refs. [5] and [35].

triaxial minimum with $\beta=0.6$ and $\gamma \approx 16^{\circ}$ for the $0^{+}$state. The energy gain due to the restoration of rotational symmetry in the ground state of ${ }^{24} \mathrm{Mg}$ in the present investigation is about $5 \mathrm{MeV}$. The minimum on the PES for the $0^{+}$state has a larger deformation $\beta_{p}=0.56$ close to the data $\beta=0.57[34]$. Keeping in mind the strong pairing gaps found in our meanfield calculations, the introduction of PNP in our calculations is not expected to introduce great changes in the results.

The right panel of Fig. 2 shows that there is a minimum in the PES for the $2^{+}$state localized at $\beta \approx 0.55, \gamma \approx 10^{\circ}$. Using the 3DAMP+RMF-PC approach we calculate the spectrum of ${ }^{24} \mathrm{Mg}$ based on this triaxial mean-field state. This is the only way to obtain $K=2$ bands in our calculation. In Fig. 3 we show this spectrum and compare it with 
the known experimental data. The observed energies are qualitatively reproduced by the theory, even though the calculated levels are systematically higher in energy, which might be due to the fact that all the excited states are projected from the same mean-field state. The projected energy spectrum would be much more compressed with the inclusion of a cranking term in the mean-field calculation [16, 20, 21]. This is a strong indication that, for a quantitative understanding of the spectra, the variation should be carried out after the projection. Of course this goes beyond the present computer capabilities.

In conclusion, we have studied the properties of the ground state and low-lying excited states in ${ }^{24} \mathrm{Mg}$ using a full three dimensional angular momentum projection on top of a relativistic mean-field calculation based on a point-coupling Lagrangian (3DAMP+RMFPC) with monopole pairing. Ground state properties are found to be reproduced rather well with this approach, even though no pronounced minimum with obvious triaxial deformation is present. A minimum with $\beta \approx 0.55, \gamma \approx 10^{\circ}$ has been found on the PES of the first $2^{+}$state, on top of which the experimentally observed excitation energies and the $\mathrm{B}(\mathrm{E} 2)$ transition probabilities can be qualitatively reproduced. However, the predicted spacing between the levels is overestimated by this approach. It is expected that this can be cured by the introduction of a cranking term at the mean-field level. These results hint that a full GCM approach is needed for a full understanding of the properties of low-lying states in ${ }^{24} \mathrm{Mg}$, and work towards this goal is in progress.

\section{ACKNOWLEDGMENTS}

Helpful discussions with D. Vretenar are gratefully acknowledged. This research has been supported by the Asia-Europe Link Project [CN/ASIA-LINK/008 (094-791)] of the European Commission, the National Natural Science Foundation of China under Grant No. 10775004, 10221003, 10720003, 10705004, the Bundesministerium für Bildung und Forschung, Germany under project 06 MT 246 and by the DFG cluster of excellence "Origin and Structure of the Universe" (www.universe-cluster.de).

[1] Parikh J 1968 Phys. Lett. B 26607

[2] Lombard R et al 1970 J. de Phys. Supp. C 290

[3] Endt P M, Van Der Leun C 1978 Nucl. Phys. A 3101 
[4] Meyer M A et al 1972 Nucl. Phys. A 185625

[5] Branford D et al 1975 Nucl. Phys. A 241349

[6] Ring P and Schuck P 1980 The Nuclear Many-Body Problem, Springer, Heidelberg

[7] Grammaticos B 1975 Nucl. Phys. A 25290

[8] Girod M and Grammaticos B 1983 Phys. Rev. C 272317

[9] Bonche P et al 1987 Nucl. Phys. A 467115

[10] Oi M 2005 Phys. Rev. C 72057304

[11] Koepf W and Ring P 1988 Phys. Lett. B 212397

[12] Hirata D et al 1996 Nucl. Phys. A 609131

[13] Valor A et al 2000 Nucl. Phys. A 671145

[14] Rodriguez-Guzman R R et al 2002 Nucl. Phys. A 709201

[15] Niksic T et al 2006 Phys. Rev. C 73 034308; 2006 Phys. Rev. C 74064309

[16] Hara K et al 1982 Nucl. Phys. A 38514

[17] Burzynski K, Dobaczewski J 1995 Phys. Rev. C 511825

[18] Enami K et al 1999 Phys. Rev. C 59135

[19] Hayashi A et al Phys. Rev. Lett. 53337

[20] Baye D and Heenen P H 1984 Phys. Rev. C 291056

[21] Zdunczuk H et al 2007 Phys. Rev. C 76044304

[22] Bender M et al 2008 nucl-th/arXiv:0805.4383.

[23] Serot B D and Walecka J D 1986 Adv. Nucl. Phys. 16 1; 1997 Int. J. Mod. Phys. E 6515

[24] Ring P 1996 Prog. Part. Nucl. Phys. 37193

[25] Vretenar D et al 2005 Phys. Rep. 409101

[26] Meng J et al 2006 Prog. Part. Nucl. Phys. 57470

[27] Bürvenich T et al 2002 Phys. Rev. C 65044308

[28] Bender M et al 2000 Eur. Phys. J. A 859

[29] Onishi N and Yoshida S 1966 Nucl. Phys. 80367

[30] Balian R and Brezin E 1969 Nuovo Cimento 6437

[31] Neergard K, Wüst E 1983 Nucl. Phys. A 402311

[32] Yao J M et al 2008 in prepare

[33] Audi G et al 2003 Nucl. Phys. A 729337

[34] Fewell M P et al 1979 Nucl. Phys. A 319214 
[35] Keinonen J et al 1989 Nucl. Phys. A 493124 